\title{
Amplitude integrated EEG 3 and 6 hours after birth in full term neonates with hypoxic-ischaemic encephalopathy
}

\author{
M C Toet, L Hellström-Westas, F Groenendaal, P Eken, L S de Vries
}

\begin{abstract}
Aim-To assess the prognostic value of amplitude integrated EEG (aEEG) 3 and 6 hours after birth.

Methods-Seventy three term, asphyxiated infants were studied (from two different centres), using the Cerebral Function Monitor (CFM Lectromed). The different aEEG tracings were compared using pattern recognition (flat tracing mainly isoelectric (FT); continuous extremely low voltage (CLV); burstsuppression (BS); discontinuous normal voltage (DNV); continuous normal voltage (CNV)) with subsequent outcome.

Results-Sixty eight infants were followed up for more than 12 months (range 12 months to 6 years). Twenty one out of 68 infants $(31 \%)$ showed a change in pattern from 3 to 6 hours, but this was only significant in five cases $(24 \%)$. In three this changed from BS to CNV with a normal outcome. One infant showed a change in pattern from CNV to FT and had a major handicap at follow up. Another infant showed a change in pattern from DNV to BS, and developed a major handicap at follow up. The other 16 infants did not have any significant changes in pattern: 11 infants had CLV, BS, or FT at 3 and 6 hours and died $(n=9)$ in the neonatal period or developed a major handicap $(n=2)$. Five infants had a CNV or DNV pattern at 3 and 6 hours, with a normal outcome. The sensitivity and specificity of $\mathrm{BS}$, together with FT and CLV, for poor outcome at 3 hours was 0.85 and 0.77 , respectively; at 6 hours 0.91 and 0.86 , respectively. The positive predictive value (PPV) was $78 \%$ and the negative predictive value (NPV) $84 \% 3$ hours after birth. At 6 hours the PPV was $86 \%$ and the NPV was $91 \%$.

Conclusion-aEEG could be very useful for selecting those infants who might benefit from intervention after birth asphyxia.

(Arch Dis Child Fetal Neonatal Ed 1999;81:F19-F23)
\end{abstract}

Keywords: cerebral function; amplitude integrated EEG; hypoxic-ischaemic encephalopathy; birth asphyxia

The incidence of hypoxic-ischaemic encephalopathy (HIE) has remained constant over the past four decades $(2-9 / 1000) .^{1}$ Therapeutic intervention to reduce delayed neuronal death or programmed cell death following birth asphyxia may become possible within the next few years. ${ }^{2-9}$ The exact duration of the so-called therapeutic window is unknown, but is considered to be only a few hours (2-6 hours) after birth. ${ }^{4}{ }^{10}$ Because treatment may have serious side effects, only those infants should be enrolled who might benefit from the treatment. Early and accurate identification is therefore of great importance.

In this respect, the predictive value of early parameters, such as fetal heart monitoring, umbilical artery acidaemia, Apgar scores, or the combination of all three, has been reported as being very limited. ${ }^{11-15}$ Important predictors of an adverse outcome, which are available in the first hours, were delayed onset of breathing $>10$ minutes, administration of chest compression at birth, and seizures. Based on predicted probabilities of $>0.50$, the sensitivity of the predictive model was $85 \%$, with a specificity of $68 \%$ at 60 minutes of age. ${ }^{15} 16$

Imaging techniques, such as cranial ultrasound scans and magnetic resonance imaging (MRI), are useful for prognosis, but not until 24 hours or more after birth. ${ }^{17-19}$ Neurophysiology, evoked potentials, ${ }^{20}{ }^{21}$ and the more commonly used EEG, are extremely useful for the first 24 hours. ${ }^{22-26}$ Amplitude integrated EEG (aEEG) is a user friendly technique, based on pattern recognition, that can be used in high risk neonates. ${ }^{27}$ aEEG is increasingly being used in neonatal intensive care units, and has a very high concordance with standard EEG. ${ }^{28-33}$ In a previous study we showed that aEEG and evoked potentials were the most useful techniques for predicting outcome within 6 hours of life after birth asphyxia. ${ }^{33} 34$ Considering the short therapeutic window after birth asphyxia, even six hours might be too late for optimal intervention. We therefore tried to establish the prognostic value of the aEEG within three hours of delivery.

\section{Methods}

Seventy three term infants (gestational age $37-42$ weeks) were studied (38 infants from the Wilhelmina Children's Hospital, Utrecht, the Netherlands, and 35 infants from University Hospital, Lund, Sweden). They were admitted to neonatal intensive care within three hours of birth and were treated for perinatal asphyxia between January 1987 and September 1997 (18 of the Lund infants were included in the previous study by Hellström-Westas et $a l^{33}$ ). Informed parental consent was obtained in all cases.

The children were diagnosed as having asphyxia when they met at least three of the following criteria: 
(A)

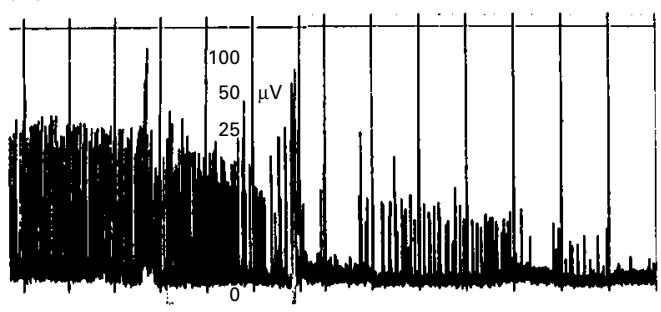

(C)

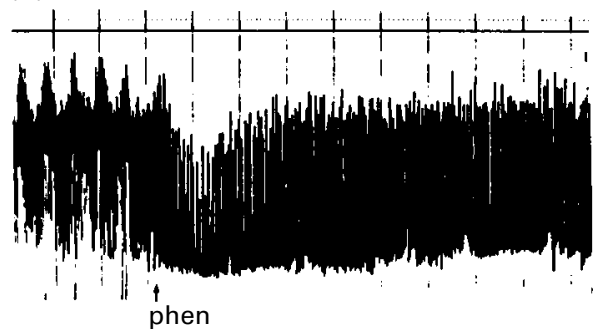

(B)

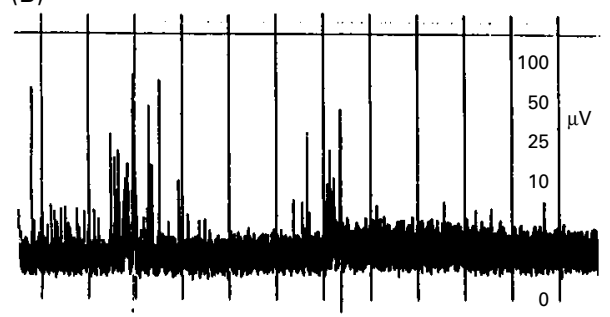

(D)

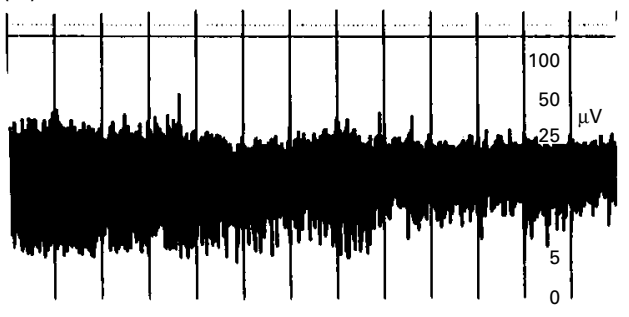

Figure 1 Examples of patterns obtained with the Cerebral Function Monitor. (A) BS pattern changing to an FT pattern; (B) CLV pattern; (C) epileptic activity (sawtooth pattern) changing to BS pattern after phenobarbitone; (D) DNV pattern changing to $C N V$ pattern.

- signs of intrauterine asphyxia, as indicated by late decelerations on fetal monitoring or by meconium staining of the amniotic fluid;

- arterial cord blood $\mathrm{pH}<7.10$;

- delayed onset of spontaneous respiration;

- Apgar score of $<5$ at 5 minutes;

- multi-organ failure

After admission an amplitude integrated continuous EEG recording (aEEG) (Cerebral Function Monitor, CFM 4640, Lectromed, Devices Ltd, UK) was applied by the attending neonatologist. The aEEG records a single channel EEG from biparietal electrodes. The filtered signal is rectified, smoothed, and amplitude integrated before it is written out at slow speed $(6 \mathrm{~cm} /$ hour $)$ at the cotside. ${ }^{30-32}$

We looked at different aEEG patterns using pattern recognition ${ }^{30}{ }^{33}$ :

Flat tracing (FT): Very low voltage, mainly inactive (isoelectric) tracing with activity below $5 \mu \mathrm{V}$.

Continuous extremely low voltage (CLV): Continuous background pattern of very low voltage (around or below $5 \mu \mathrm{V}$ ).

Burst-suppression (BS): Discontinuous background pattern; periods of very low voltage (inactivity) intermixed with burst of higher amplitude.

Discontinuous normal voltage (DNV): Discontinuous trace, where the voltage is predominantly above $5 \mu \mathrm{V}$.

Continuous normal voltage (CNV): Continuous activity with voltage $10-25(-50) \mu \mathrm{V}$.

Epileptic activity was also identified.

Table 1 Flat tracing mainly isoelectric (FT); continuous extremely low voltage (CLV); burst-suppression (BS); discontinuous normal voltage (DNV); continuous normal voltage $(C N V)$

\begin{tabular}{|c|c|c|c|c|c|c|c|c|c|c|}
\hline \multirow[b]{2}{*}{ Outcome } & \multicolumn{5}{|c|}{ aEEG at 3 hours } & \multicolumn{5}{|c|}{ aEEG at 6 hours } \\
\hline & $F T$ & $C L V$ & $B S$ & $D N V$ & $C N V$ & $F T$ & $C L V$ & $B S$ & $D N V$ & $C N V$ \\
\hline Died & 1 & 5 & 15 & & 1 & 7 & 5 & 10 & & 1 \\
\hline Major handicap & & 1 & 5 & 1 & 1 & 2 & 1 & 4 & & \\
\hline Global delay & & & 1 & & 2 & & & 1 & & 2 \\
\hline Normal & & & 8 & 5 & 22 & & & 5 & 2 & 28 \\
\hline
\end{tabular}

Examples of CFM patterns are given in fig 1.

The traces were analysed off line by two investigators and subsequently analysed by one investigator from the other centre. Interobserver agreement was reached in all but one of the cases. This case was excluded from the study; 73 infants were included in the study.

In 35 infants (16 in Utrecht and 19 in Lund) phenobarbitone with or without diazepam had been administered before the aEEG recording was started. In most cases this medication was given as prophylaxis after birth asphyxia, ${ }^{26}{ }^{35}$ or if babies needed to be intubated.

The survivors were seen in the follow up clinic at 3, 9,18, 24 months, 3 and 5 years of age. Assessment of outcome was made using the Griffiths mental developmental scale and items from the Amiel-Tison and Grenier evaluation and Touwens test. ${ }^{36-38}$ The Alberta Infant Motor Scale (AIMS) was also used in children younger than 18 months of age. ${ }^{39}$

Cerebral palsy was classified according to the criteria of Hagberg et al..$^{40}$ Adverse outcome was defined as death in the neonatal period, cerebral palsy, or global delay (Developmental Quotient $<80$ on the Griffiths scale $\geqslant 12$ months).

For estimation of predictive ability, the sensitivity, specificity, and positive predictive values and negative predictive values of different items were calculated. To compare previous findings, ${ }^{33}$ we also estimated the efficiency (\%) as the total number of correctly predicted individuals in relation to the total number of observations made (true positive + true negative /all $\times 100$ ) .

\section{Results}

Sixty eight infants were followed up for more than 12 months (range 12 months to six years). The aEEG pattern 3 and 6 hours after birth, together with the outcome, are shown in table 1 and figs 2 and 3. 
Infants with normal outcome

aEEG at 3 hours

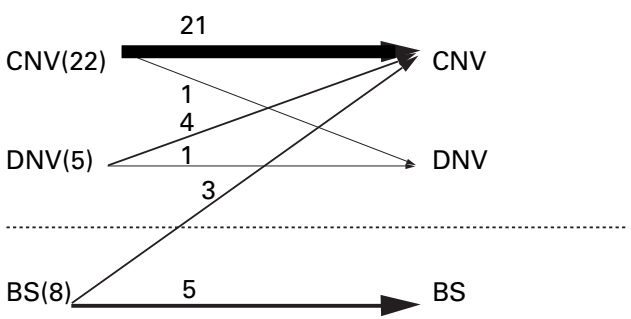

CLV

CLV

FT

FT

Figure 2 aEEg patterns in infants with normal outcome.

The sensitivity and specificity for BS, together with FT and CLV for poor outcome (cerebral palsy or death) at three hours and six hours, are given in table 2 . The positive predictive value (PPV) was $78 \%$ and the negative predictive value (NPV) was $84 \% 3$ hours after birth. At 6 hours the PPV was $86 \%$ and NPV was $91 \%$. The efficiency was $81 \%$ and $88 \% 3$ and 6 hours, respectively.

Twenty one out of $68(31 \%)$ infants showed a change in pattern between 3 and 6 hours. Improvement occurred in seven cases: in three this changed from $\mathrm{BS}$ to $\mathrm{CNV}$, with a subsequent normal outcome. In four infants the pattern changed from DNV to CNV, with a normal outcome in all infants.

In 14 cases deterioration was noted: in one infant the pattern changed from CNV to DNV, with a subsequent normal outcome. One infant showed a change in pattern from DNV to BS, and developed a major handicap. One infant showed a change in pattern from CNV to FT and had a major handicap at follow up. The other 11 infants did not have a significant change in pattern; they either had CLV, BS, or FT at 3 and 6 hours and died $(n=9)$ in the neonatal period or developed a major handicap $(\mathrm{n}=2)$.

The change in pattern between 3 and 6 hours was therefore significant only in the three cases who improved from BS to CNV, and the two who deteriorated (CNV to FT and DNV to BS). According to clinical criteria for HIE defined by Sarnat, ${ }^{41} 27$ infants developed mild encephalopathy (grade I); 17 developed moderate encephalopathy (grade II); and 20 developed severe encephalopathy (grade III). The degree of encephalopathy could not be classified in four infants. The aEEG patterns at 3 hours and the degree of encephalopathy are listed in table 3.

Thirty three infants $(47 \%)$ received antiepileptic medication before the aEEG recording started: 31 infants received a loading dose of phenobarbitone (seven in combination with midazolam or diazepam) and two infants
Infants with poor outcome

aEEG at 3 hours

aEEG at 6 hours

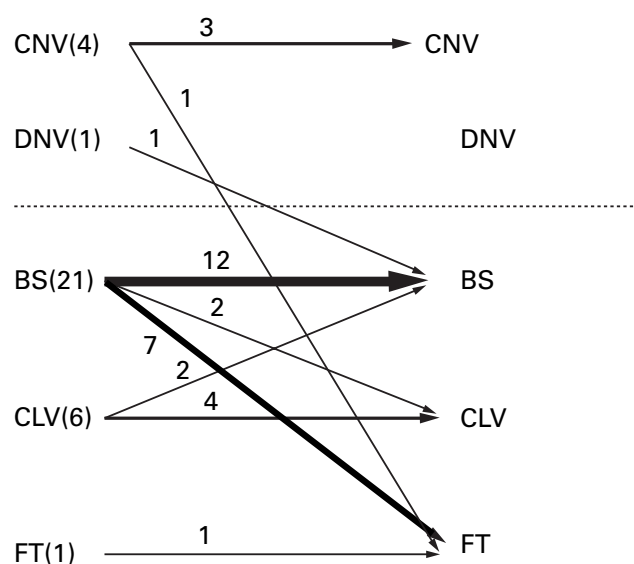

Figure 3 aEEG patterns in infants with poor outcome.

received diazepam alone. The background activity of the aEEG at three hours was classified as CNV in five infants; DNV in two; BS in 20 infants; CLV in five infants; and FT in one infant. Only three of these infants had a significant improvement in background activity between 3 and 6 hours.

In 24 infants (35\%) anti-epileptic drugs were administered between 3 and 6 hours (phenobarbitone with or without phenytoin or clonazepam; one patient received lidocaine). In 14 cases the background pattern did not change between 3 and 6 hours: CNV in three infants; BS in nine; CLV in one infant; and FT in one. The other infants $(n=10)$ did not show a significant change in background pattern. In one infant the pattern changed from CNV to DNV; in three the pattern changed from DNV to CNV; BS changed to FT or CLV in five infants; and in one infant the pattern changed from CLV to BS.

Table 2 Predictive values (\%) of BS together with FT and CLV for poor outcome

\begin{tabular}{llll}
\hline $\begin{array}{l}\text { Predictive value } \\
\text { (FT,BS,CLV/poor outcome) }\end{array}$ & Combined & Utrecht & Lund \\
\hline At 3 hours & & & \\
Sensitivity & 85 & 89 & 80 \\
Specificity & 77 & 75 & 79 \\
PPV & 78 & & \\
NPV & 84 & & \\
Efficiency & 81 & & \\
At 6 hours & & 89 & 93 \\
Sensitivity & 91 & 81 & 89 \\
Specificity & 86 & & \\
PPV & 86 & & \\
NPV & 91 & & \\
Efficiency & 88 & & \\
\hline
\end{tabular}

Table 3 aEEG patterns and clinical criteria for hypoxic ischaemic encephalopathy (HIE)

\begin{tabular}{lccc}
\hline aEEG at 3 hours & $\begin{array}{c}\text { HIEI } \\
(n=27)\end{array}$ & $\begin{array}{c}\text { HIE II } \\
(n=17)\end{array}$ & $\begin{array}{c}\text { HIE III } \\
(n=20)\end{array}$ \\
\hline CNV & 22 & 4 & \\
DNV & 3 & 2 & \\
BS & 2 & 10 & 14 \\
CLV & & 1 & 5 \\
FT & & & 1 \\
\hline
\end{tabular}


Epileptic activity was already seen at 3 hours after birth in nine infants. Their background activity was: CNV in one infant; $\mathrm{DNV}$ in one; BS in six; and CLV in one.

Three infants had epileptic activity 3 hours after birth in spite of a previous loading dose of phenobarbitone. Six of the 24 infants who received anti-epileptic drugs between 3 and 6 hours still had epileptic activity 6 hours after birth.

In $59(80 \%)$ a standardised EEG was performed in the first week of life: in 34 cases within the first 24 hours; in 13 cases between 24 and 48 hours. In general, there was good agreement between the aEEG and the standardised EEG. These data will be reported separately.

Eighteen of the 20 infants with a grade III encephalopathy and three of the 17 infants with a grade II encephalopathy died in the neonatal period. Intensive care was withdrawn when a combination of neurophysiology (aEEG, standard EEG, evoked potentials (SEP, VEP)), and imaging (ultrasound, MRI) remained severely abnormal on two or more occasions. Six infants died within 24 hours; five infants died within 48 hours; the other infants $(n=10)$ died between the third and the seventh day after birth.

\section{Discussion}

Previous studies have already shown that aEEG can predict outcome accurately at 6 hours of age following perinatal asphyxia..$^{33} 34$ Both studies showed a PPV of $86 \%$ and $84 \%$, respectively, and an NPV of $96 \%$ and $91 \%$, respectively. This agrees with earlier standard EEG studies recorded within the first week of life.$^{22-25}$

We were able to show that aEEG already has a good predictive value at 3 hours of age following perinatal asphyxia. This has major implications when selecting those infants who could benefit from postasphyxial intervention.

CNV pattern $(n=26)$ at 3 hours was almost invariably a good prognostic indicator of normal outcome. Four infants still had a CNV pattern at 3 hours despite a previous loading dose of phenobarbitone. One infant died in the neonatal period, due to a severe intracranial haemorrhage. This child already showed epileptic activity at 3 and 6 hours. Another child developed a major handicap; this infant deteriorated very rapidly and had an FT at 6 hours of age. One infant, who comes from a very poor social background, showed global delay.

DNV pattern $(n=6)$ also seemed to be a good predictor of normal outcome, but the number of cases in this group is still limited. Most infants $(n=4)$ had developed a CNV pattern by 6 hours. One infant still had a DNV pattern at 6 hours, with a subsequent normal outcome. In only one infant did the pattern deteriorate and change to BS; this infant developed a major handicap. FT $(n=9)$ and CLV (n $=8$ ) at 3 or 6 hours carried a poor prognosis. All but four infants died in the neonatal period; the other four developed cerebral palsy.
The most interesting cases were those with a BS pattern at 3 hours $(n=29)$. Twenty infants had already received anti-epileptic medication (a loading dose of phenobarbitone $(20 \mathrm{mg} / \mathrm{kg}$ ) with or without midazolam or diazepam). This might have affected the background pattern at 3 hours. We have already shown that depression of background activity following administration of anti-epileptic medication depends on the severity of brain damage. In infants with only HIE the effect only lasts 30-60 minutes, showing a more discontinuous pattern, while a change to a BS pattern can be seen in cases with severe HIE. ${ }^{33}{ }^{34}$ As four of the 26 cases with a CNV on admission had also received anti-epileptic medication, the BS pattern was unlikely to have been due to the medication alone. Only three of the infants with a BS pattern significantly improved background activity between 3 and 6 hours, with a subsequent normal outcome. All three had previously received a loading dose of phenobarbitone. In the first few years of this study, the anti-epileptic drugs were given as a prophylaxis after birth asphyxia. ${ }^{26} 33$

Five out of 17 infants with a BS pattern at 3 and 6 hours had a normal neurodevelopmental outcome. In these cases the background pattern normalised within 24 hours.

Although changes in aEEG pattern were seen in 21 of the $68(31 \%)$ cases, these changes were only important in five $(24 \%)$. Three infants improved from a BS to a CNV pattern, with a subsequent normal outcome, and two deteriorated, with a subsequent poor outcome (CNV to FT and DNV to BS).

In this study three infants would not have been given treatment as they had a CNV or DNV pattern at 3 hours. Two infants deteriorated very rapidly and developed a major handicap. The other infant died, due to a severe intracranial haemorrhage. In eight infants treatment would have been given, but this would not have been necessary. They had a BS pattern at 3 hours and a subsequent normal outcome. Five of them still had a BS pattern at 6 hours.

In the previous study by Hellström-Westas et al, early seizures were also associated with a poor outcome. ${ }^{33}$ In this study seizures 3 hours after birth were associated with a poor outcome in five out of nine cases, while four had a normal outcome. On the other hand, absence of seizure activity on aEEG 3 hours after birth was not predictive of a normal neurodevelopmental outcome. The number of cases with very early seizures, clinical or aEEG-seizures, was relatively small. It was therefore difficult to confirm previous data. ${ }^{1516}$

Hellström-Westas et $a l^{33}$ also studied the prognostic ability of aEEG during the first three hours of birth, but only in a small number of infants with an efficiency of $74 \%$. With a larger number of infants, we found an efficiency of $81 \%$ three hours after birth. In the study where different non-invasive techniques were compared, the aEEG had the highest positive predictive value $(84.2 \%)$ and negative predictive value $(91.7 \%)$ six hours after birth. ${ }^{34}$ Six hours after birth we found about the same 
positive predictive value of $86 \%$ and a very similar negative predictive value of $91 \%$. Again, the positive predictive value at 3 hours $(78 \%)$ turned out to be lower than the negative predictive value $(84 \%)$. Interestingly, there was good agreement between the predictive values obtained in both centres.

Assessment of neurodevelopmental outcome was slightly different in the two centres and the infants were still young ( $>12$ months) when last seen in the follow up clinic. Adverse outcome was taken as death, cerebral palsy, or global delay $(\mathrm{DQ}<80)$. So far a global delay was found only in three infants (with one infant from a poor social background), but it is quite possible that a higher number will be found when the infants are seen again at a later stage. Further follow up of these infants is in progress and they will be seen again at 3 and 5 years of age.

The aEEG gives a long term, cotside, on-line, trend recording of cerebral electric activity, readily available at any time of the day. Although it does not give information about localisation, changes in background activity and seizure activity are easy to identify.

Postnatal evaluation after perinatal asphyxia using aEEG can reliably predict neurodevelopmental outcome, as early as 3 hours after birth. Intervention and treatment following perinatal asphyxia may not be free of risk. ${ }^{10}$ aEEG could be useful for selecting those infants who might benefit from early intervention after perinatal asphyxia, and because of its high negative predictive value $(84 \%)$, avoid unnecessary risks related to treatment for those infants that do not need intervention.

Dr Paula Eken was supported by a grant from the Prinses Beatrix Fonds (No 92-2372)

We thank the Axel and Margaret Axison Johnson foundation and Lund University Hospital funds for their support.

This article is dedicated to Professor N W Svenningsen.

1 Hagberg B, Hagberg G, Olow I. The changing panorama of cerebral palsy in Sweden. VI. Prevalence and origin during

2 Levene M, Blennow M, Whitelaw A, Hanko E, Fellman V, Hartley R. Acute effects of two different doses of magnesium sulphate in infants with birth asphyxia. Arch Dis Child 1995;73:F174-7.

3 van Bel F, Shadid M, Moison RMW, et al. Effect of Allopurinol on postasphyxial free radical formation, cerebral hemodynamics, and electrical brain activity. Pediatrics 1998;101:185-93.

4 Haaland K, Loberg EM, Steen PA, Thoresen M. Posthypoxic hypothermia in newborn piglets. Pediatr Res 1997;41:505-12.

5 Amess PN, Penrice J, Cady EB, et al. Mild hypothermia after severe transient hypoxia-ischaemia reduces the delayed rise in cerebral lactate in the newborn piglet. Pediatr Res 1997;41:803-8.

6 Thoresen M, Penrice J, Lorek A, Cady EB, Wylezinska M, Kirkbride V. Mild hypothermia after severe transient hypoxia-ischaemia ameliorates delayed energy failure in the newborn piglet. Pediatr Res 1995;37:667-70.

7 Gunn TR, Gunn AJ, Gluckman PD. Substantial neuronal rescue with prolonged selective head cooling begun $5 \mathrm{hrs}$ after cerebral ischeamia in fetal sheep. Pediatr Res 1997;41:898A

8 Edwards AD, Azzopardi D. Hypothermic neuronal rescue treatment: from laboratory to cotside? Arch Dis Child 1998;78:F88-F91.

9 Edwards AD, Wyatt JS, Thoresen M. Treatment of hypoxic brain damage by moderate hypothermia. Arch Dis Child 1998;78:F85-F8

10 Vannucci RC, Perlman JM. Interventions for perinatal hypoxic-ischaemic encephalopathy. Pediatrics hypoxic-ischaemic

11 Carter S.B, McNabb F, Merenstein G.B. Prospective validation of a scoring system for predicting neonatal mor-
bidity after acute perinatal asphyxia. $\mathcal{f}_{\text {Pediatr }}$ bidity after acute
12 King TA, Jackson GL, Josey AS, Vedro DA, Hawkins H, Mchugh Burton K. The effect of profound umbilical artery acidemia in term neonates admitted to a newborn nursery. F Pediatr 1998:132:624-9.

13 Shankaran S. Identification of term infants at risk for neonatal morbidity. F Pediatr 1998;132:571-2.

14 Dijxhoorn MJ, Visser GHJ, Huisjes HJ, Fidler V, Touwen BJC. The relation between umbilical $\mathrm{pH}$ values and neurological morbidity in full term appropriate for date infants. Early Hum Dev 1985;11:33-42.

15 Ekert P, Perlman M, Steinlin M, Hao Y. Predicting the outcome of postasphyxial hypoxic-ischemic encephalopathy within 4 hours of birth. F Pediatr 1997;131:613-17.

16 Wayenberg JL, Vermeylen D, Bormans J, Magrez P, Muller MF, Pardou A. Diagnosis of severe birth asphyxia and early prediction of neonatal neurological outcome on term asphyxiated newborns. F Perinat Med 1994;22:129-36.

17 Eken P, Jansen GH, Groenendaal F, Rademaker K, de Vries LS. Intracranial lesions in the fullterm infant with hypoxic ischaemic encephalopathy: ultrasound and autopsy correlation. Neuropediatrics 1994;25:301-7.

18 Rutherford MA, Pennock JM, Dubowitz LMS. Cranial ultrasound and magnetic resonance imaging in hypoxic ischaemic encephalopathy: a comparison with outcome. Dev Med Child Neurol 1994;36:813-25.

19 Martin E, Barkovich AJ. Magnetic resonance imaging in perinatal asphyxia. Arch Dis Child 1995;72:F62-F70

20 De Vries LS. Somatosensory evoked potentials in term neonates with postasphyxial encephalopathy. Clin Perinatol 1993;20:463-82.

21 Gibson NA, Graham M, Levene MI. Somatosensory evoked potentials and outcome in perinatal asphyxia. Arch Dis Child 1992;67:393-9.

22 Holmes G, Rowe J, Hafford J, Schmidt R, Testa M, Zimmerman A. Prognostic value of the electroencephalogram in neonatal asphyxia. Electroencephalogr Clin Neurophysiol 1982;53:60-72.

23 Pezzani C, Radvyani-Bouvet MF, Relier JP, Monod N. Neonatal encephalography during the first 24 hours of life in fullterm newborn infants. Neuropediatrics 1986;17:11-18.

$24 \operatorname{Monod}$ N, Pajot N, Guidasci S. The neonatal EEG: statistical studies and prognostic value in fullterm and preterm babies. Electroencephalogr Clin Neurophysiol 1972;32:529-44.

25 Watanabe K, Miyazaki S, Hara K, Hakamada S. Behavioral state cyclus, background EEGs and prognosis of newborns with perinatal hypoxia. Electroencephalogr Clin Neurophysiol 1980;49:618-25.

26 Svenningsen NW, Blennow G, Lindroth M, Gäddlin PO, Ahlstrom A. Brain-oriented intensive care treatment in severe neonatal asphyxia. Arch Dis Child 1982;57:176-83.

27 Murdoch-Eaton D, Toet M.C, Livingston J, Smith I, Levene $M$. Evaluation of the cerebrotrac 2500 for monitoring of cerebral function in neonatal intensive care. Neuropediatrics 1994;5:122-8.

28 Arbald F, Verma UL, Tejani NA. Cerebral Function Monitor in the neonate. 2: Birth asphyxia. Dev Med Child Neurol 1984;26:162-8.

29 Bjerre I, Hellström-Westas L, Rosen I, Svenningsen NW. Monitoring of cerebral function after asphyxia in infancy. Arch Dis Child 1983;58:997-1002.

30 Thornberg E, Thiringer K. Normal patterns of cerebral function monitor traces in term and preterm neonates. Acta Paediatr Scand 1990;79:20-5.

31 Hellström-Westas L. Comparison between tape-recorded and amplitude-integrated EEG monitoring in sick newborn infants. Acta Paediatr Scand 1992;81:812-19.

32 Prior PF, Maynard DE. Monitoring cerebral function. Longterm recordings of cerebral electric activity and evoked potentials. Amsterdam: Elsevier, 1986.

33 Hellström-Westas L, Rosen I, Svenningsen NW. Predictive value of early continuous amplitude integrated EEG recording on outcome after birth asphyxia in full term recording on outcome after birth asphyx
infants. Arch Dis Child 1995;72:F34-F8.

34 Eken P, Toet M.C, Groenendaal F, de Vries L.S. Predictive value of early neuroimaging, pulsed Doppler and neurophysiology in full term infants with hypoxic-ischaemic encephalopathy. Arch Dis Child 1995;73:F75-80.

35 Hall RT, Hall FK, Daily DK. High-dose phenobarbital therapy in term newborn infants with severe perinatal asphyxia: a randomized, prospective study with three-year asphyxia: a randomized, prospective
follow-up. 7 Pediatr 1998;132:345-8.

36 Griffiths R. The abilities of babies: a study in mental measurement. Amersham: Association of Research in Infant and Child Development, 1976

37 Amiel-Tison C, Grenier A. Evaluation neurologique du nouveau-né et du nourisson. Paris: Masson, 1980.

38 Touwen BLC. Examination of the child with minor neurological dysfunction. In: Clinics in Developmental Medicine 71. London: William Heinemann Medical Books, 1979 .

39 Piper MC, Darrah J. Motor assessment of the developing infant. Philadelphia: WB Saunders Co, 1994

40 Hagberg B, Hagberg G, Olow I. The changing panorama of cerebral palsy in Sweden. I. Analysis of general changes. Acta Paediatr Scand 1975;64:187-92.

41 Sarnat HB, Sarnat MS. Neonatal encephalopathy following fetal distress. A clinical and electroencephalographic study. Arch Neurol 1976;33:696-705. 